\title{
Change: From Industry 4.0 to Society 5.0 -Taking the comparison of related development strategies between Germany and Japan as an example
}

\author{
Kang, Jiali ${ }^{1, a}$ \\ ${ }^{1}$ The Institute for Social and Cultural Research (ISCR), Macao University of Science \& Technology, \\ Taipa, Macao SAR, China \\ ame@mail.im
}

Keywords: Development strategy; Industrial policy; Social governance; Comparative study

\begin{abstract}
One of the current performances of the competition among major powers in the world is the competition of development strategy focus on the future. Therefore, in recent years, they have formulated corresponding development strategies to try to occupy the commanding heights. This article takes the recently released national development strategies of Germany and Japan as an example, combines the similar strategies formulated by major global powers in the 1980s, and sets out the comparability of the strategies of Germany and Japan, and discusses the background of the two proposals. Compared with the differences in background, trend, context, starting point, and national conditions, and conclude that any country's development strategy needs to follow the general rules of human social and the inspiration of both those two countries' strategies.
\end{abstract}

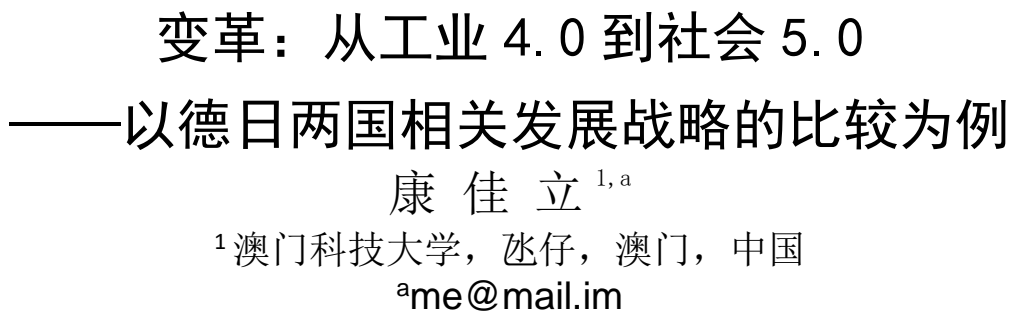

关键词：发展战略；产业政策；社会治理；比较研究

摘 要: 当前世界各主要大国间的竞争, 表现之一就是着眼于未来的发展战略竞争, 于是它 们在近几年纷纷制定了相应的发展战略来试图占领制高点。本文以德国和日本最近发布的国 家发展战略为例, 结合上世纪八十年代全球主要大国制定的类似战略为铺垫, 从德日两国战 略具有的可比性出发, 论及两者提出的背景, 并从背景、走向、脉络、出发点以及国情等诸 方面比较两者的不同, 最后得出任何一国的发展战略都需要遵循人类社会发展的一般规律以 及两国战略带给人的启发。

\section{1. 引言}

21 世纪第一个十年过后，随着德国率先提出 “工业 4.0”的大战略规划，打破了世界大 国间长期在制定发展战略领域的静默期。全球尚且具有一定影响力的国家，纷纷不甘落后制 定了本国的未来战略规划。这其中就有美国先进制造业战略、韩国制造业创新 3.0 战略、英 国高科技创新战略、新工业法国和中国创新制造 2025, 以及其后提出的日本社会 5.0 计划等。 这些战略的提出, 再现了上世纪 80 年代间各主要大国纷纷提出战略规划的浪潮, 对一国此类 战略的攻击在一定程度上成为掣肘该国的手段 ${ }^{(1)}$ 。由其后所带来的影响轨迹可知，新世纪的新 战略势必对国家内部发展和国家间实力的对比带来影响，因此需要切实的加以重视。

\footnotetext{
(1) 参考《又进一步! 美国公布对中国产品加征关税建议清单, 挑明剑指 “中国制造 2025”》, 来源环球网: http://world.huan
} 


\section{2. 两者提出的背景}

工业 4.0 (Industry 4.0) 最先是由德国人提出的概念。它是基于制造业的国家战略性规划, 是强化产业竞争力、实现产业变革的有益尝试。该战略以智能制造为核心，以信息技术革命 为突破口, 辅以治理的革命, 反映了工业经济数字化、信息化、智能化融汇成网络化发展的 新潮流，标志着全球快速进入全新网络时代治下的工业新经济形态，形成了巨大的影响力。 契合于西方国家的政治习惯，工业 4.0 战略首先由民间的德国工业研究联盟发起，其重要性 在得到官方层面的认可后上升为国家战略，并成为德国政府力推的《德国高技术战略 2020》 十大未来项目之一，获得了政府部门先期的大额资助 ${ }^{(1)}$

面对世界各国相继制定了国家层面的战略规划，日本不甘落后并适时提出 “社会 5.0” 概 念, 这是源于日本学术界认为人类即将进入所谓的 “超智能社会”。鉴于此, 在 2016 年 1 月 日本内阁会议通过的 “第五期（2016 2020 年度）科学技术基本计划” 正式提出了基于 “超 智能社会” 的 “社会 5.0”（Society 5.0）概念，并得到了该国上至官方下至民间的社会各界积 极响应, 并在 5 月底颁布的《科学技术创新战略 2016》中对其做了进一步阐释。这个概念是 对人类社会发展进程必然出现的虚拟空间与现实空间高度融合的预见为前提, 对人类社会带 来巨大变革的前瞻性概括为基础, 期望通过在此领域的优先布局从而达到占领制高点, 确保 国家主体的持续性优先发展为目标的一项规划。日本政府认识到其重要性, 很快把它作为与 德国“工业 4.0”类似的政策方针提升到国家战略的层面。

\section{3. 比较的具体内容}

首先，工业 4.0 和社会 5.0 是德日两国现阶段在产业发展上面临危机的大背景下提出的。 [1]具体表现在除属于自然界的资源环境、生存健康等挑战外, 还包括属于人类社会以及以因 特网为主的人造虚拟网络内部间各国的激烈冲突。这主要体现在金融危机的重创、国家间各 种软硬实力竞争的加剧和全球性不确定因素挑战日益严峻等方面。其中, 发达国家自身在产 业升级换代上面临尾大不掉的困境, 以及后发国家在科学技术上对先进国家的追赶, 甚至其 它先发国家对某一发达国家形成的夹击均为这类问题的突出表现。于是为了保持自身优势, 需要相应进行策略上的规划与调整，方便对政府的大政方针政策进行宏观指导。

其次，两国在制定各自大战略的走向上不尽相同。回溯上世纪八十年代中期，包括刚刚 改革开放的中国在内, 世界各主要大国同样掀起了一轮类似今天这样的, 以科学探索和新技 术革命为主线的国家层面战略制定浪潮。以至于在几年的时间里，以美国的 “星球大战计划” 为先导, 接连出现了欧洲的 “尤里卡计划”、日本的 “人类新系统研究计划”、中国的 “863 计划”等一系列国家战略规划指引。

其中日本的 “人类新系统研究计划” 和现今的 “社会 5.0 ” 战略一以贯之，均是单一国别 主体下的战略性指引。它一如既往的彰显了日本民族中对强者的膜拜和充分学习的特征, 使 得该计划与美国的 “星球大战” 计划在大胆、前卫甚至浮夸上如影随形。从今天看来, 这个 计划相当前瞻的关注到人工智能在未来发展中对人类的作用和影响, 并力图开创在此领域的 研究高地占据主动。只是与现阶段对人工智能的研究主要处于不对人体本身进行改造, 而是 围绕网络技术和脑科学, 仅限于通过外界工具充当桥梁进行研究的学科不同, “人类新系统研 究计划” 十分先觉的, 甚至是含有 “黑科学” 意味的选择了生物技术的大方向来对人类进行 全方位革新性的探索甚至改造。小到个体的分子、细胞, 大到人体的构成和系统的循环, 都 全方位被笼络进该计划的研究范围中。时至今日，该计划在诸多方面仍旧十分超前。

事实上，与“社会 5.0 ”所提出的背景类似, “人类新系统研究计划”也有其现实考量。 在强化国家综合实力, 寻找新的经济增长点, 提升国民生活质量, 解决经济与社会矛盾, 特

qiu.com/exclusive/2018-04/11767441.html。

(1) 据悉获得了德国联邦教育局及研究部和联邦经济技术部的联合资助, 投资预计达 2 亿欧元。来源: 物联中国, http://www.50cnnet.com/show-39-131456-1.html。 
别是试图解决 “少子化” 问题上如出一辙。这也体现在两者均被学者推荐采用 “官、产、学” 相结合的组织体制上。不过亦不能排除跟风的原始作用。毕竟作为当时 GDP 常年保持全球第 二的经济强国日本，在周遭诸国均订立了一些列国家发展长远规划的大前提下，也需要制定 类似计划来彰显该国在引领世界潮流上从来就不甘落后。直到今天, 仍然适用于新近提出的 “社会 5.0 ”计划。

然而一连串现实打击让日本人不得不重新审视自身的未来。特别是面临严重少子化的问 题，让近几十年来从民间到官方都对该问题予以特别重视，甚至专门设置“少子化担当大臣” 这一职位, 来专项关注并处理这一问题及给日本带来的深刻社会危机。只是回过头来可以看 到, 从 “人类新系统研究计划” 开始到三十多年后的 “社会 5.0 ” 计划, 在外国人 “归化” 问 题上向来持保守态度等多方面原因导致的日本 “少子化” 危机, 非但没有得到解决甚至还有 愈演愈烈的趋势(1)这从另外一个层面可以证实 “人类新系统研究计划” 的实施效果, 并对后 来“社会 5.0 ”的结局提供一个相对真实的参考意见。于是可以得出日本在本世纪头二十年里, 完全没有体现出对 “人类新系统研究计划” 有所研究的学者所认为的那样, 让 “21 世纪, 有 可能成为日本的世纪” [2]。

相对于德国而言，“尤里卡计划”更像是一个宏观层面跨越国界的开放性规划，并打下了 相当的时代烙印。在上世纪 80 年代中期, 西欧主要国家联合起来对抗美苏两国争霸的格局占 据主流, 它要求其着重强调西欧的振兴和团结。不过这种观念导致的是欧共体及其后欧盟各 国合作制定的具体规划, 由于各国国情不同理念差距过大, 出现了复杂的利益纠纷导致协调 困难所造成的延误和内耗。它严重干扰了各项计划的顺利实施，这在欧洲版的 GPS 工程一一 “伽利略计划”上体现得尤为明显。而欧盟扩大后新加入的原社会主义阵营国家在观念和经 济发展水平上的差距, 更是加重了这一趋势。此外, 某些域外具有巨大影响力的超级大国, 处于自身原因对欧洲完整性的担忧 ${ }^{2}$, 结合欧洲国家内部近些年来的治理和战略失误对自身造 成的负面影响 ${ }^{3}$ ，又离间并破坏了欧洲的团结与协作。

因此现今欧洲各国单独制定具体规划的现状，根源上不排除是因为欧盟主体完整性在英 国脱欧后带来了巨大变化，欧盟作为单一整体没有上世纪的 “流行”。同时相对一个松散联盟 所造成协调上的困难, 一国内部制定适合国情的战略规划对自身更具积极影响。于是直到当 前, 欧洲各国重拾自主制定国家战略规划的方针, 不再过分强调欧盟这一政治实体凌驾于国 家之上，从一方面证明了它们走过的弯路与当今世界格局和时代潮流相悖，德国亦不例外。

再次, “工业 4.0” 和 “社会 5.0” 具有较大的相似性, 着重表现在两者所秉持的战略脉络 上。前者是按照普遍认可的工业革命进程来递进式推演。从最早的水利和蒸汽为主要动力来 开发机械制造的第一次工业革命, 到以电能驱动并基于劳动力分工形成大工业化的第二次工 业革命, 再到如今以电子信息技术为主要引领的自动化技术为第三次工业革命, 然后逐步推 移到最后预见的以物理信息系统 (Cyber-Physical System, 简称 CPS) 为主要驱动模式的人类 下一代工业革命, 并开展战略性的谋划。与此类似, “社会 5.0” 则是按照人类社会的发展进 程为主要依据, 汲取 “工业 4.0” 的思考体系, 提出了从最原始的狩猎社会开始, 依序把人类 社会划分为狩猎社会、农耕社会、工业社会和信息社会这四个存在的阶段, 直到最后预见的 “超智能社会”阶段。

不过 “社会 5.0” 还是 “工业 4.0” 的继承与发扬。最明显的是两者发布的时间先后顺序 在名称上的体现由 “4.0” 变为 “5.0”。除了照其解释的按照工业发展和社会进程来排序取名 外，从侧面也反映了后者对前者的吸收革新带来的升级换代。正如历史上日本对德国的诸多 “学习” 和 “跟随” 类似, 两个战略的提出具有塑造新时代 “轴心” 形象的雏形。其中, 日

\footnotetext{
(1) 参考《日本 1920 年-2060 年人口动态分布图》得出的结论。来源: 日本国立社会保障 - 人口问题研究所, http://ww4.sinaimg.cn/large/be6bdb92jw1f1xeasstnsg20m50ea7wh.gif。以及搜狐网: 《日本应对少子化收效极为有限》: http://www.sohu.com/a/40575661_113727。

(2) 例如美国前总统小布什把欧洲划分为 “新欧洲” 和 “老欧洲” 等。

3) 席卷欧洲的难民问题亦为其重要表现之一，甚至是英国 “脱欧” 的重要催生因素。
} 
本的 “社会 5.0 ” 已然在吸取前者的经验上, 并不局限于工业的范畴, 把整个战略体系置于更 宏大的人类社会大视角下来观察，甚至不惜把 “工业 4.0 ” 的核心概念一一工业革命作为自身 战略的一个步骤进行阐述, 独辟蹊径的由其扩展到农业、防灾减灾、健康及社会管理等诸多 新领域, 最终促成整个社会的有序进步。当然, 这也可以认为日本是 “主动回避” 德国具有 强大实力的工业领域来扬长避短。

第四, 两个战略的出发点基本一致但方向不同。它们都是力图维系自身国力辅以解决国 民在当前和未来相当长时期内面临的困难为前提。均是以网络技术在新时代新环境下的新应 用为基础, 着重强调物联网 ( IOT)、大数据、人工智能（AI）等前沿网络技术的普及和运用。 两者均是置身于包括 “人一网络” 在内的 “实体一虚拟” 相结合的大框架之下。只是落实到 具体发展方向上德国多立足工业, 竭尽所能的发挥自身在产业制造与技能上的领先优势, 由 此认为通过持续不断的工业化可以实现和解决人类面临的几乎所有困境, 从而保障自身的先 进性。其中，德国在 “工业 4.0” 中除了熟练运用被人熟知的因特网（internet）、物联网（IoT） 外, 还提及了服联网（Internet of Services, IoS)、人联网（和 Internet of People, IoP) 等全新 概念。 [3]事实上，日本提出的 “社会 5.0” 概念比 “工业 4.0” 所涵盖的范围要大得多，在政 策实施和细节处理上也相应困难。于是日本优先侧重于人类社会的构成上, 通过有机整合社 会各个子系统发挥最大效益, 以所谓的精准服务为切入点的指导方针, 来面对社会日益增加 的细分化需求。这种认知和对社会理解的革命性变化, 让日本经济产业联合会直接把 “社会 $5.0 ”$ 提升到日本的第四次产业革命的高度上来 ${ }^{(1)}$

最后, 国情的不同对两者影响巨大。日本面临的严重内需问题在 “少子化” 和 “高龄化” 问题上体现得尤为明显, 甚至明确提出要以保护国民健康为基础来订立 “健康立国” 的国策 [4]。这个现实注定日本需要以社会的大格局来制定政府的宏观政策。于是会以提升社会质量 为中心的 “社会 5.0” 为前提，任何产业的更新换代不能摆脱为其服务的局面。德国则完全不 同。在制定相关 “工业 4.0” 计划伊始，德国就毫不犹豫认为自己的 “制造业是世界上最具竞 争力 “之一', (特别是) 装备领域的制造已然成为全球的领导者”, 这是由于德国 “能够掌控 复杂的生产过程。该过程是由不同地区承担不同任务的众多合作者共同完成”, 同时让德国“完 全有必要巩固并发展其在装备制造产业上的领军地位”，[5]遂引入一种潜在的产业形态

“工业 4.0”，把其作为德国 “2020 高技术战略” 确定的十大未来项目之一，来具体落实在气 候和能源、健康和营养、交通、安全和通讯五大领域, 并提出各自的行动计划和措施。[6]

这种不同点也体现在各国的社会习俗上。在对 “社会 5.0” 相关政策的解读中, 日本经济 产业联合会认为无可避免的需要打破 “五墙” ( 5 つの壁, Five Walls) 这一条块化的现象, 来 防止对新经济形式和社会形态造成不可预见的阻碍乃至破坏性的改变。首当其冲的是来自官 方层面的 “墙” ——相关政府机构的各自为政造成的分化即 “内耗”。其解决办法应该在国家 政策的制定上, 注重 “官、产、学” 相结合, 并使政府的推动集约化, 以及建立跨界别的咨 议机构等。这对与日本具有相似文化基因的我国而言, 在官方层面具有相当的指导意义和警 醒作用。而在文化完全不同的德国, 在无根基和土壤的情况下尚且无法理解东方思维, 官方 对于政策的制定和推动则采取国家层面统一化的机制, 即加持者的角色, 而非“麻烦制造者”。

\section{4. 结论和启发}

基于人们惯常的认知，科技革命和工业文明对于社会发展与变迁是不能被忽视的因素， 这是冲突对社会变革的体现。只是一国所提出的政策对科技发展的促进作用, 并力图由此加 快社会进步, 引导其往更优方向变迁的美好理想, 在现实影响下多有不尽如制定者初衷的过 程和结局。

(1) 资料来源: http://www.sohu.com/a/114817175_463971。 
在狩猎社会和农耕社会阶段人类以生存为出发点和目标, 因此主要体现在自然界与人类 社会之间的冲突。它让维持生存的技术得以长足进步，但仍旧与低下的生产力相伴而生。

其后的工业社会和信息社会两个阶段，人们在基本解决了生存问题后，迎来了更高层次

发展的冲突。这种冲突主要体现在人类社会和诸如因特网在内的人造网络之间，是人类 能动性的充分反映和体现。特别是出现了基于二次创造与生产的人造网络创新, 从理论上让 人类的生产力水平得到无限的发展。这是以持续引入前沿科学为先导, 开创性的让其和技术 在辩证的基础上展开融合, 极大的促进了生产力的发展, 使得人们获得了不仅仅满足于存在 的更高阶段的生活质量和享受，并竭力通过合理的发展方式让其不断扩展并延续下去。

“工业 4.0”战略的提出恰好契合了人类发展的这一态势, 受到了不仅限于提出国的重视, 甚至形成了一系列的标准, 引领了世界潮流。它重点提出了解决冲突的着力点, 并尝试找出 解决办法, 为人类社会进入全新的工业文明时代提供了一个前瞻性的建议。在这点上人类社 会是一个整体, 均置于这一具有普世性的宏观体系之下, 得到了一干国家的认可并积极效仿。 因此可以简单的概括, “工业 4.0 ” 是积极采取先进的智能技术来解决人类发展进程中核心部 分的产业发展问题。

“社会 5.0” 这一概念本质上是把冲突过后的稳定作为目标，是冲突发展的必然结果。其 手段是力图使用最先进的智能技术来解决人类发展的社会问题。这种稳定性由于社会的发展 程度和文明因素的不同千差万别，带来了相对 “工业 4.0” 而言的特殊性导致的局限性。同时 “社会 5.0” 选择的是不同冲突主体间逐步融合的方向来提出概念，通过融合各种子社会重塑 一个全新的母社会，来对单个社会的劣势进行补充，从整体上发挥比个体更大的优势。

只是任何政策实施的成败与否，除了政策本身以外，还面临许多不可预料因素的影响。 除了政策对领域内尚且预备一定的预测性，体系外保障政策的顺利执行甚至反哺，除了对制 定者是一个能力上的考验, 对执行者更是一个技术上的检验。相对德国已经完善提出并制定 了详细的 “工业 4.0” 战略规划, 日本 “社会 5.0” 的提出显得物忙和不得已为之。从第三方 角度来看, 除了显示自身能够继续处于引领世界潮流的优势地位外, 以此增加国民的国家荣 誉感和民族自豪感的成分更大。毕竟, 事关长远和全局的 “社会 5.0” 被日本寄予厚望来改变 自身面临的困境，可是这一社会难题是不是仅凭科技就能解决让人怀疑，甚至还会指向一国 的政治制度这样更深的层面。

\section{References}

[1] Ministry of Education, Culture, Sports, Science and Technology (MEXT), Japan, White Paper on Science and Technology 2016,http://www.mext.go.jp/b_menu/hakusho/html/hpaa201601/detail/1374226.htm.

[2] F. Yu, W. Bo, and Z. Heng, Rebuilding the Basis of Future Development for Japan's Economic and Social--An Analysis of Japan's "Human New System Research Program", Future \& Development, 1988.

[3] Federal Ministry of education and research, Germany, Recommendations for implementing the strategic initiative INDUSTRIE 4.0_Final Report of the Industrie 4.0 Working Group,pp.24.

[4] Cabinet Office, Japan, Comprehensive Strategy on Science, Technology and Innovation 2016,http://www8.cao.go.jp/cstp/sogosenryaku/2016.html.

[5] Federal Ministry of education and research, Germany, Recommendations for implementing the strategic initiative INDUSTRIE 4.0_Final Report of the Industrie 4.0 Working Group,pp.5-13.

[6] Die Bundesregierung, Ideen. Innovation. Wachstum. Hightech-Strategie 2020 für Deutschland, http://www.hightech-strategie.de/index.php. 